\title{
Normobaric hypoxia overnight impairs cognitive reaction time
}

\author{
Stephan Pramsohler ${ }^{1 *} \mathbb{D}$, Stefan Wimmer ${ }^{1,2}$, Martin Kopp ${ }^{2}$, Hannes Gatterer², Martin Faulhaber ${ }^{2}$, \\ Martin Burtscher ${ }^{2}$ and Nikolaus Cristoph Netzer ${ }^{1,2,3}$
}

\begin{abstract}
Background: Impaired reaction time in patients suffering from hypoxia during sleep, caused by sleep breathing disorders, is a well-described phenomenon. High altitude sleep is known to induce periodic breathing with central apneas and oxygen desaturations, even in perfectly healthy subjects. However, deficits in reaction time in mountaineers or workers after just some nights of hypoxia exposure are not sufficiently explored. Therefore, we aimed to investigate the impact of sleep in a normobaric hypoxic environment on reaction time divided by its cognitive and motoric components. Eleven healthy non acclimatized students ( $5 f, 6 \mathrm{~m}, 21 \pm 2.1$ years) slept one night at a simulated altitude of $3500 \mathrm{~m}$ in a normobaric hypoxic room, followed by a night with polysomnography at simulated $5500 \mathrm{~m}$. Preexisting sleep disorders were excluded via BERLIN questionnaire. All subjects performed a choice reaction test (SCHUHFRIED RT, S3) at $450 \mathrm{~m}$ and directly after the nights at simulated 3500 and $5500 \mathrm{~m}$.

Results: We found a significant increase of cognitive reaction time with higher altitude $(p=0.026)$. No changes were detected in movement time ( $p=$ n.s.). Reaction time, the combined parameter of cognitive- and motoric reaction time, didn't change either ( $\mathrm{p}=$ n.s.). Lower $\mathrm{SpO}_{2}$ surprisingly correlated significantly with shorter cognitive reaction time $(r=0.78, p=0.004)$. Sleep stage distribution and arousals at $5500 \mathrm{~m}$ didn't correlate with reaction time, cognitive reaction time or movement time.

Conclusion: Sleep in hypoxia does not seem to affect reaction time to simple tasks. The component of cognitive reaction time is increasingly delayed whereas motoric reaction time seems not to be affected. Low $\mathrm{SpO}_{2}$ and arousals are not related to increased cognitive reaction time therefore the causality remains unclear. The fact of increased cognitive reaction time after sleep in hypoxia, considering high altitude workers and mountaineering operations with overnight stays, should be further investigated.
\end{abstract}

Keywords: Reaction time, Hypoxia, Cognition, Sleep, Extreme altitude

\section{Background}

With the popularity of mountaineering even ill-prepared climbers tend to spend their nights during ascent at elevated altitudes $[1,2]$. Climbing projects get more audacious, driven by excessive ambition and overestimation [3]. Also working operations at high altitude are still rising. This includes work at constructions or in mines, touristic enterprises and research work in observatories

\footnotetext{
*Correspondence: s.pramsohler@gmx.net; hypoxieleiter@hermann-buhl-hypoxie.de

1 Hermann Buhl Institute for Hypoxia and Sleep Medicine Research, Ghersburgstr. 9, 83043 Bad Aibling, Germany

Full list of author information is available at the end of the article
}

[4]. This requests a more detailed look at the consequences of high altitude sleep and successive performance of tasks.

It is well known that sleep quality decays increasingly with severe hypoxia. Especially periodic breathing and central apneas seem to disturb restorative sleep [5-10]. While ascending, alpinists and altitude workers experience their lowest oxygen levels during the night when arbitrary breathing does not happen. Therefore, symptoms of acute mountain sickness are likely to appear immediately after sleeping at a new altitude [11]. Several studies show that exposure to hypoxic environments affects reaction time [12-18]. Prolonged 
reaction to critical tasks at altitude could enhance the risk of injury. Reaction time is formed by a cognitive and a motoric part where the cognitive or "premotor" part seems to play a significant role [19]. It seems that from data provided about trauma and death during mountaineering, deficits in cognition form a considerable part. Between 1926 and 2006 1.3\% of the attempts to reach the summit of Mount Everest ended fatal. From 113 deaths during this time, trauma is the most reported cause. Narrations from their previous health status suggest that the most common symptoms leading to this were profound fatigue and cognitive impairment [20].

If sleep in hypoxic condition causes a momentary impairment in reacting to simple tasks, this could be a cause for slow or wrong actions. More knowledge might be important not only for preventing accidents in mountaineers, but also in the above mentioned workers at high altitude.

The aim of this study is to investigate reaction time divided by its cognitive and motoric aspect in a standardized environment after sleep in high and extreme altitude. By using normobaric hypoxia, standardized conditions without environmental and constitutional influences are ensured.

\section{Methods}

\section{Subjects}

Five female and six male healthy students (mean age $21.0 \pm 2.1$ years) participated in the study. All Subjects have been recruited from the sports faculty of the University of Innsbruck and were very sportive. Subjects reporting any medical disorder, including sleep apnea (Berlin Questionnaire), smoking and pre-acclimatization to high altitude were excluded from participation.

\section{Protocol}

According to Dykiert et al. [21], simple reaction time starts to be significantly impaired in altitudes over $4000 \mathrm{~m}$. A normobaric room using an oxygen expulsion System (Low Oxygen Systems; Berlin-Buch, Germany) was used for the simulation. This allows reducing oxygen in the whole chamber to a minimum of $9.3 \%$ while keeping $\mathrm{CO}_{2}$ levels correspondingly low. As stated by Saugy et al. normobaric hypoxia demands a higher simulation to provoke similar effects as in hypobaric environments [22]. Since only a single exposure night in normobaric hypoxia was used, it seemed necessary to simulate $5500 \mathrm{~m}$ to provoke adequate reactions. In order to provide enough safety for the subjects and to be able to monitor a longer sleeping phase on $5500 \mathrm{~m}$, a night at simulated $3500 \mathrm{~m}$ seemed necessary for pre-acclimatization.

Eleven subjects were asked to sleep for three successive nights in an altitude chamber. After every night a reaction testing (RT Schuhfried) was performed to assess motoric and cognitive reaction time. The testing took place directly after awakening, still inside the hypoxic room. Three different heights were chosen to determine changes in cognitive and motoric reaction time after sleep. The baseline testing took place at $450 \mathrm{~m}$ above sea level, corresponding to an inspiratory oxygen fraction $\left(\mathrm{FiO}_{2}\right)$ of $20.93 \%$ (altitude at Bad Aibling, Germany). The second testing took place at a simulated altitude of 3500 m, equivalent to an $\mathrm{FiO}_{2}$ of $14,29 \%$ followed by night three at a simulated altitude of $5500 \mathrm{~m}\left(\mathrm{FiO}_{2}=9905 \%\right)$. All testing's were conducted with respectively two subjects at the time. The study protocol is displayed in Fig. 1.

Every testing night began at 11:00 p.m. with the entering of the altitude room. Night one and night two were interrupted at $6: 30$ by the investigator, to perform

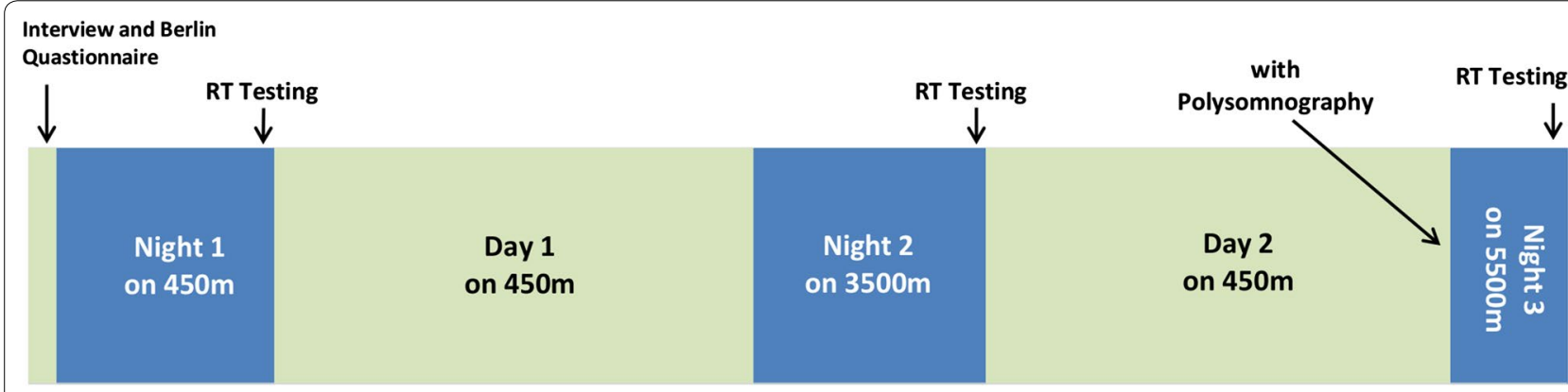

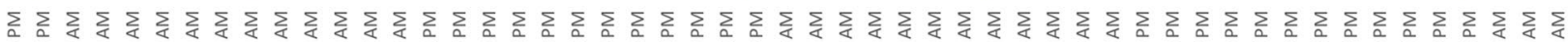

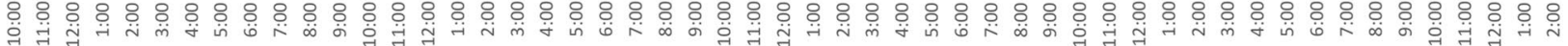
Daytime

Fig. 1 Study protocol. Study design with markings at every testing point. Wake and sleep phases correspond to the actual circadian periods of measurement (mean individual sleeping time at Night 1 and Night $2=7.50 \mathrm{~h}$, Night $3=3.23 \mathrm{~h}$ ) reaction testing is displayed as RT testing 
the reaction testing, marking the end of hypoxic exposure. All subjects were still sleeping by then. Night three was monitored by a full 12 channel polysomnography. Polysomnoghraphy was mainly chosen as a monitoring tool for safety reasons. This way it was possible to react immediately if needed. Additionally, it allows looking for influencing factors regarding altitude sleep and changes in cognitive and motoric reaction. In night three polysomnoghraphic data was used to determine actual sleeping time instead of purporting a fix sleeping interval. The subjects were instructed to be aware of the symptoms of high altitude sickness. The Lake Louise Score was assessed in a standardized manor, directly after completing the testing sequence. In case of unbearable mountain sickness symptoms, subjects were free to disrupt the night sleep at any time. If sleep was interrupted, the choice reaction test was still completed directly after awakening and still in hypoxic conditions.

The protocol was designed to exclude the influence of constitutional differences after an exhaustive hike. The aim was to exclude environmental influences and therefore isolate the effect of sleep. Normobaric hypoxia was chosen to provide more safety for the personnel and the subjects. This way, they could leave the hypoxic rooms at any time without passing through a lock.

\section{Measuring}

The reaction test (RT Schuhfried) measures the response time after both, a visual stimulus and a sound signal (Test form S3). The subjects were instructed to react as quickly as possible to the shown stimulus. The test score evaluates reaction time divided by its cognitive and motoric part needed to the respond to the shown stimulus (React when a yellow and a black dot are displayed while a sound is played simultaneously). Subjects were asked to press and hold the "SPACE" button on the keyboard during the whole testing time. When a relevant signal was displayed they were instructed to move to the "ENTER" key and back to the "SPACE" key as fast as possible. The elapsed time from the showing of the stimulus until leaving the "SPACE" button was measured as cognitive reaction time. The time needed for the movement from the "SPACE" key to pressing the "ENTER" key was measured as motoric reaction time. Obviously, the reaction time is the sum of motoric- and cognitive reaction time. The testing time was $10 \mathrm{~min}$. Each subject was instructed by two learning phases each lasting $2 \mathrm{~min}$. The twelve channel polysomnography on simulated $5500 \mathrm{~m}$ was carried out according to the American Academy of Sleep Medicine standard of 2007. Acute mountain sickness symptoms were only assessed orally.

\section{Data analysis}

Data are presented as medians or means (were applicable) \pm standard deviation (SD). Data analyses were performed with the SPSS statistical software package (PASW Statistics for Windows version 21.0, SPSS Inc., Chicago, IL, USA). An ANOVA for repeated measurements was applied to identify differences between the different heights. The Pearson test was used to determine correlations. Significance level was set at $\mathrm{p}<0.05$. A post hoc power analysis was performed with GPower 3.1.

\section{Results}

We have seen a significant increase of cognitive reaction time with an increase in altitude as shown in Fig. 2 $(\mathrm{p}=0.026)$. At $450 \mathrm{~m}$ median cognitive reaction time was $0.5 \mathrm{~s} \pm 0.047$ and increased from $0.54 \mathrm{~s} \pm 0.05$ at $3500 \mathrm{~m}$ up to $0.56 \mathrm{~s} \pm 0.07$ at $5500 \mathrm{~m}$. The main statistical effects were detected between 450 and $3500 \mathrm{~m}$ as well as from 450 to $5500 \mathrm{~m}$. No changes were detected in motoric reaction time $(450 \mathrm{~m}=0.24 \mathrm{~s} \pm 0.14$, $3500 \mathrm{~m}=0.29 \mathrm{~s} \pm 0.06$ and $4500 \mathrm{~m}=0.26 \mathrm{~s} \pm 0.74$, $\mathrm{p}=$ n.s.). The superordinate parameter of reaction time, didn't change as well and was measured as $0.77 \mathrm{~s} \pm 0.17$ at $450 \mathrm{~m}, 0.82 \mathrm{~s} \pm 0.08$ at $3500 \mathrm{~m}$ and $0.8 \mathrm{~s} \pm 0.79$ at $5500 \mathrm{~m}$ ( $\mathrm{p}=$ n.s. $)$.

All subjects reported sleeping problems at $3500 \mathrm{~m}$ and quite severe periodic breathing episodes were measured on $5500 \mathrm{~m}$ simulated altitude with great individual variance. Male subjects reacted more distinct, i.e. had more apneas with increasing hypoxia than females (Mean Apnea/hypopnea index AHI, respiratory events/h: male $=121.65 \pm 62.84$, female $=59.59 \pm 68.13, \mathrm{p}=$ n.s.). No statistically significant gender differences could be detected.

At an altitude of $5500 \mathrm{~m}$ individual mean peripheral oxygen saturation $\left(\mathrm{SpO}_{2}\right)$ readings showed a positive correlation towards shorter cognitive reaction time $(\mathrm{r}=0.78, \mathrm{p}=0.004)$. Low individual mean oxygen saturation was associated with a faster cognitive reaction (Fig. 3). Polysomnographic parameters show a severely impaired sleeping behavior at simulated $5500 \mathrm{~m}$ (Table 1). The parameter of AHI correlates with total sleeping time $(\mathrm{r}=0.57, \mathrm{p}=0.003)$ as well as sleep phase $3(\mathrm{r}=0.45$, $\mathrm{p}=0.022$ ) and rapid eye movement sleep (REM) periods $(\mathrm{r}=0.54, \mathrm{p}=0.004)$. Individuals who sleep longer have a significantly higher AHI, especially if they have more phase 3 and REM sleep (Table 1). A tendency regarding $\mathrm{SpO}_{2}$ and total sleeping time could be detected as higher $\mathrm{SpO}_{2}$ allows longer sleep or vice versa $(r=0.55, p=0.079)$. The post hoc power analysis for the parameter cognitive reaction time accounted for an effect size of 0.97 . 
Sec.

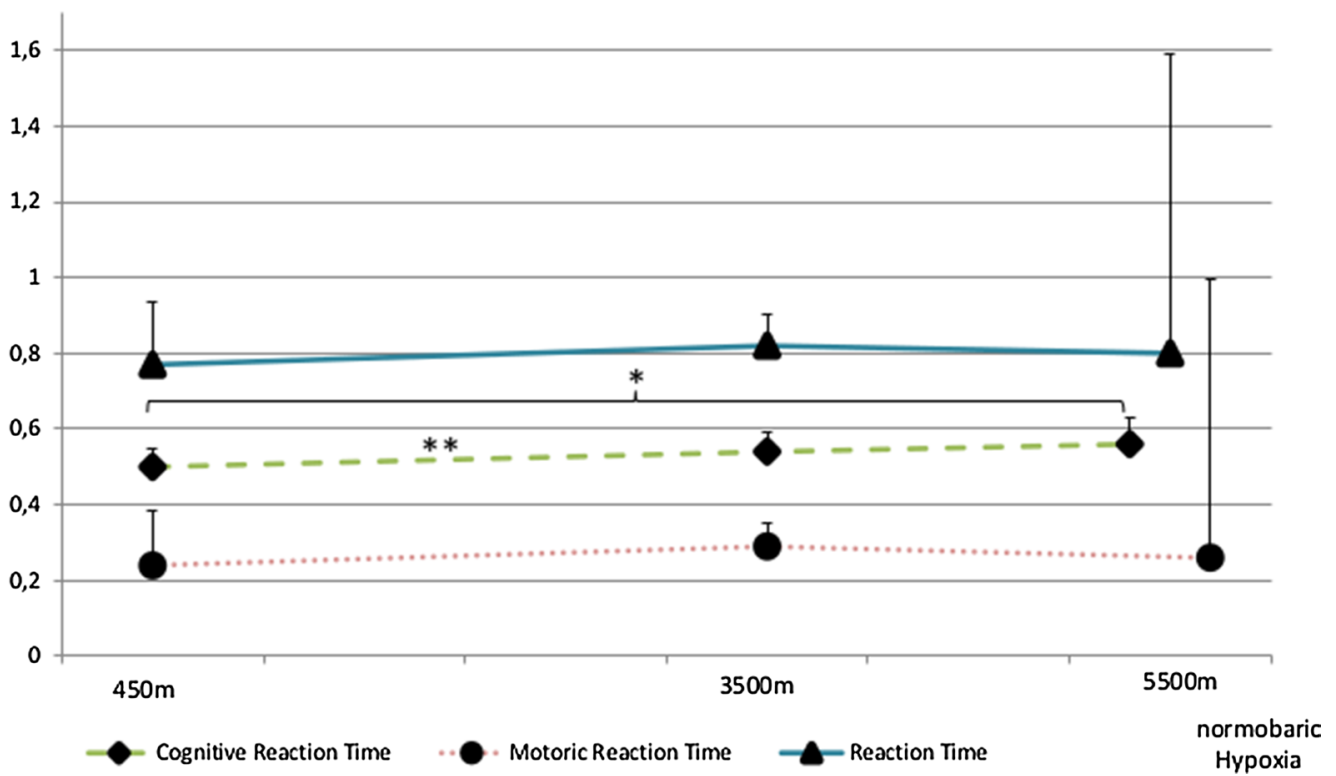

Fig. 2 Changes in reaction time and its components, cognitive- and motoric reaction time over the three testing altitudes. Cognitive reaction time is prolonged with increasing altitude. Significant changes have been marked as follows $\left({ }^{*} p<0.05 ;{ }^{* *} p<0.025\right)$. Data is displayed as medians with standard deviation; reaction time and its components are displayed in seconds $(\mathrm{s})$ and altitude in simulated meters a.s.l (m)

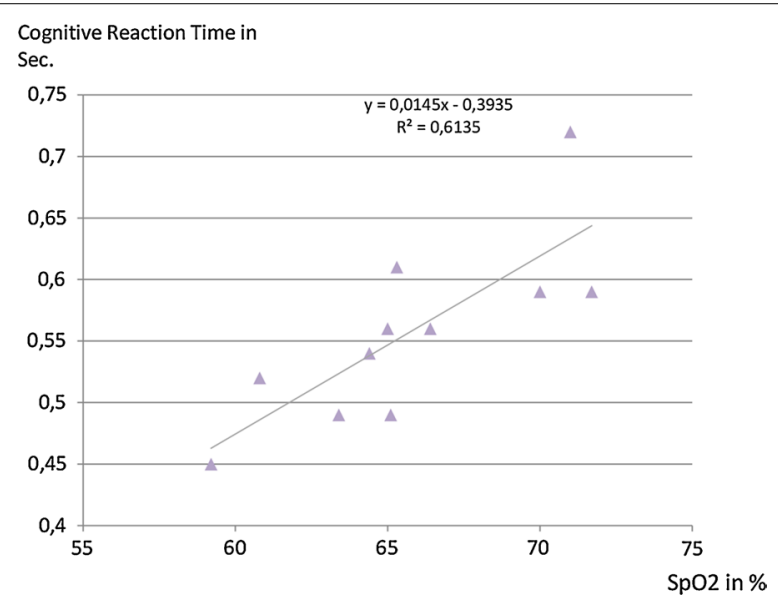

Fig. 3 Correlation between cognitive reaction time and mean $\mathrm{SpO}_{2}$ overnight at $5500 \mathrm{~m}$. High mean $\mathrm{SpO}_{2}$ overnight correlates with slow cognitive reaction time $(R=0.78, p=0.004)$

\section{Discussion}

To our knowledge, this is the first study on reaction time and its single components, cognitive reaction time and motoric reaction time, after sleep in normobaric hypoxia assessed in a standardized environment. Until now, most studies examined this matter in real altitude with great environmental and subjects-related, constitutional influences $[12,13]$. The greater parameter of reaction time was not affected as much by low $\mathrm{FiO}_{2}$ levels during the night. Nevertheless, data show that high altitude sleep has a significant impact on cognitive reaction time.

In the present sample, cognitive reaction time decreased constantly throughout the trial with decreasing $\mathrm{FiO}_{2}$ levels. This is in line with several authors postulating the impact of hypoxemia on cognitive functioning when sleeping in hypobaric hypoxia [12-18]. A strong promoter for cognitive dysfunction after acute hypoxia exposure of lowlanders could be the raised inflammation markers as C-Reactive Protein and Interleukin as suggested by Sheng and Goldstein [13, 23]. In contradiction to other studies, in our sample poor cognitive reaction was not associated with low peripheral $\mathrm{SpO}_{2}$ levels during the night at $5500 \mathrm{~m}$ simulated altitude. This leads us to believe that low saturation levels are not the only factor to affect cognitive reaction. Subjects with shorter total sleeping time showed a tendency towards lower $\mathrm{SpO}_{2}$ levels and during this short exposure time, less impaired sleep. Short exposure in combination with a quite light sleep may not impair cognitive functioning as much as longer periods of deeper sleep. This observation requires more research and is not sufficiently probed by our data.

Motoric reaction shows a high individual variability and changes did not reach statistical significance. As anaerobic energy systems in the skeletal muscle outbalance aerobic mechanisms in such a short reaction testing and the movement intensity is quite low, it is not 
Table 1 Polysomnographic data at simulated $5500 \mathrm{~m}$

\begin{tabular}{|c|c|c|c|c|c|c|c|c|c|c|c|}
\hline ID & C-01 & C-02 & C-03 & C-04 & C-05 & C-06 & C-07 & C-08 & C-09 & $C-10$ & $C-11$ \\
\hline $\operatorname{Sex}(f / m)$ & $\mathrm{m}$ & $f$ & $f$ & $\mathrm{~m}$ & $f$ & $\mathrm{~m}$ & $f$ & $\mathrm{~m}$ & $f$ & $\mathrm{~m}$ & $\mathrm{~m}$ \\
\hline TST (min) & 155 & 108 & 40 & 103.5 & 347 & 373.5 & 122 & 334.5 & 135.5 & 157.3 & 260.9 \\
\hline N 1 (\%) & 3.9 & 9.3 & 33.8 & 11 & 13.1 & 7.5 & 12.3 & 24.8 & 10.5 & 15.9 & 27.9 \\
\hline N 2 (\%) & 44.5 & 39.8 & 47.5 & 63.3 & 52.9 & 66.7 & 75 & 52.8 & 39.9 & 54.5 & 48.5 \\
\hline N $3(\%)$ & 37.7 & 50.9 & 18.5 & 18.8 & 20.3 & 60.5 & 12.7 & 22.4 & 41.7 & 21.6 & 19.8 \\
\hline REM (\%) & 13.9 & 0 & 0 & 6.8 & 13.7 & 9.6 & 0 & 0 & 7.7 & 7.9 & 3.7 \\
\hline$C A(n)$ & 382 & 53 & 111 & 312 & 291 & 907 & 4 & 759 & 0 & 163 & 87 \\
\hline $\mathrm{CH}(\mathrm{n})$ & 15 & 7 & 1 & 17 & 189 & 40 & 12 & 39 & 13 & 9 & 20 \\
\hline $\mathrm{AHI}(\mathrm{n} / \mathrm{h})$ & 153.68 & 33.33 & 168 & 190.72 & 83 & 152.13 & 7.87 & 143.139 & 5.76 & 65.61 & 24.61 \\
\hline HF (bpm) & 58 & 92.9 & 82.2 & 89.6 & 71.9 & 62.8 & 73.8 & 91.6 & 73.8 & 79.8 & 72.48 \\
\hline $\mathrm{SpO}_{2}(\%)$ & 66.4 & 60.8 & 65.1 & 59.2 & 71.7 & 70 & 64.4 & 65.3 & 65 & 71 & 63.4 \\
\hline
\end{tabular}

TST total sleeping time, N1 non REM 1, N2 non REM 2, N3 non REM 3, REM rapid eye movement sleep, CA central apneas, $C H$ central hypopneas, $A H I$ apnea hypopnea index, $\mathrm{HF}$ heart frequency, $\mathrm{SpO}_{2}$ peripheral oxygen saturation

surprising that motoric reaction is not prolonged significantly by low saturation levels [24].

As expected, sleep quality was severely impaired in the present study group at simulated $5500 \mathrm{~m}$ altitude. A piled occurrence of periodic breathing and a quite short mean total sleeping time of $3 \mathrm{~h}$ and 14 min were measured at $5500 \mathrm{~m}$. Progressively, impaired sleep quality with increasing altitude concurs with previous findings of various study groups [8, 10, 25]. Lower medium oxygen saturation during the night was associated with a better cognitive performance, which contradicts our expectations. Sleep related data did not show conclusive relations between saturation levels, AHI, sleeping phases and total sleeping time. Nevertheless, data leads us to believe that periodic breathing (i.e. high $\mathrm{AHI}$ ) may have a $\mathrm{SpO}_{2}$ stabilizing function as hypothesized by Küpper et al. [7]. Subjects suffering from severe periodic breathing showed a higher $\mathrm{SpO}_{2}$, a longer and deeper sleep with more periodic episodes, which supposedly provoked more severe impairment in cognitive reaction time.

The screening for gender related differences in reaction time didn't show any correlations. Non-significant differences were seen regarding AHI and total sleeping time. It is known that female hormone during menstrual cycle is an important factor in the regulation of stress-related behaviors and inflammatory signaling pathways [26, 27]. The lack of significant differences is supposedly owed to the small sample size.

Of course some restrictions influence our study outcome. As expected, many of the subjects only slept for a few hours at a simulated altitude of $5500 \mathrm{~m}$. This way, only a short period of altitude sleep remained to be analyzed by the investigators. Furthermore, due to the very bumpy sleeping behavior at simulated $5500 \mathrm{~m}$, difficulties in the evaluations of the sleeping related parameters have to be recognized. Sometimes it was difficult to divide body movement-waves and ECG-waves to identify sleeping stages. This fact could account for the inconclusive sleep data. A first effect of acclimation and ventilatory adaption during the first night could have affected the performance in the second testing. Nevertheless, this acclimatization night was necessary for safety reasons. For a complete assessment of sleep and the influence at reaction testing, 12 channel polysomnography would have been needed at night one and two as well. Since the study was conducted in normobaric environment there are less disruptive factors. Nevertheless, the study protocol allows only a restricted transfer to real altitude conditions.

\section{Conclusion}

Cognitive reaction time is impaired after sleeping in high altitude in normobaric conditions. The relations between sleep quality, low $\mathrm{SpO}_{2}$ and cognitive functioning still remain unclear and need further investigation. Cognitive impairment could be among one of the first effects caused by hypoxia as already hypothesized by Roach and colleagues and it could be one of the most fatal [28].

\section{Authors' contributions}

SP: conception and designing research study, conducting experiment, analyzing data, acquiring data, analyzing data, writing manuscript; SW: designing research study, analyzing data, revising manuscript; MK: conception of research study, revising manuscript; HG: analyzing data, revising manuscript; MF: analyzing data, revising manuscript; MB: analyzing data, revising manuscript; NN: conception and designing research study, providing material, analyzing data, writing manuscript. All authors read and approved the final manuscript.

\section{Author details}

${ }^{1}$ Hermann Buhl Institute for Hypoxia and Sleep Medicine Research, Ghersburgstr. 9, 83043 Bad Aibling, Germany. ${ }^{2}$ Department of Sport Science, University Innsbruck, Fürstenweg 185, 6020 Innsbruck, Austria. ${ }^{3}$ Division 
of Sports Medicine and Rehabilitation, Department of Medicine, University Ulm, Fürstenweg 185, Ulm, Germany.

\section{Acknowledgements}

Sincere thanks to Fabian Seifert for its technical support and assistance in conducting the measurements. Furthermore, we thank the publications fund of the University of Innsbruck for their contribution.

\section{Competing interests}

The authors declare that they have no competing interests.

\section{Availability of data and materials}

The datasets used and/or analyzed during the current study are available from the corresponding author on reasonable request.

\section{Ethics approval and consent to participate}

There was granted an exemption for approval by the Ethical Review Board of the Bavarian Physician Chamber (22.05.2014) Informed consent to participate was obtained from the participants.

\section{Publisher's Note}

Springer Nature remains neutral with regard to jurisdictional claims in published maps and institutional affiliations.

Received: 5 December 2016 Accepted: 10 May 2017

Published online: 15 May 2017

\section{References}

1. Randelzhofer P. DAV-Bergunfallstatistik 2012/13. 2013.

2. Mitgliederzahl Deutscher Alpenverein / Statistik. http://de.statista.com/ statistik/daten/studie/215943/umfrage/mitgliederzahl-des-deutschenalpenvereins/. Accessed 16 Aug 2016.

3. Mount Everest The British Story_-Speed Climbing Records. http://www everest1953.co.uk/speed-climbing-records. Accessed 16 Aug 2016.

4. ThK. German_UIAA MedCom Empfehlung Nr 15 Arbeit in Hypoxie 2012.

5. Johnson PL, Edwards N, Burgess KR, et al. Sleep architecture changes during a trek from 1400 to $5000 \mathrm{~m}$ in the Nepal Himalaya. J Sleep Res. 2010;19:148-56. doi:10.1111/j.1365-2869.2009.00745.x.

6. Nussbaumer-Ochsner Y, Ursprung J, Siebenmann C, et al. Effect of short term acclimatization to high altitude on sleep and nocturnal breathing. Sleep. 2012;35:419-23. doi:10.5665/sleep.1708.

7. Küpper T, Schöffl V, Netzer N. Cheyne stokes breathing at high altitude: a helpful response or a troublemaker? Sleep Breath. 2008;12:123-7. doi:10.1007/s11325-007-0155-5.

8. Bloch KE, Buenzli JC, Latshang TD, et al. Sleep at high altitude: guesses and facts. J Appl Physiol. 1985:2015(119):1466-80. doi:10.1152/ japplphysiol.00448.2015.

9. Rexhaj E, Rimoldi SF, Pratali L, et al. Sleep disordered breathing and vascular function in patients with chronic mountain sickness and healthy high-altitude dwellers. Chest. 2015. doi:10.1378/chest.15-1450.

10. Shogilev DJ, Tanner JB, Chang Y, et al. Periodic breathing and behavioral awakenings at high altitude. Sleep Disord. 2015;2015:279263. doi:10.1155/2015/279263.
11. Burgess KR, Johnson P, Edwards N, et al. Acute mountain sickness is associated with sleep desaturation at high altitude. Respirology. 2004;9:48592. doi:10.1111/j.1440-1843.2004.00625.x.

12. Davis JE, Wagner DR, Garvin N, et al. Cognitive and psychomotor responses to high-altitude exposure in sea level and high-altitude residents of Ecuador. J Physiol Anthropol. 2015;34:2. doi:10.1186/ s40101-014-0039-X.

13. Hu SL, Xiong W, Dai ZQ, et al. Cognitive changes during prolonged stay at high altitude and its correlation with C-reactive protein. PLoS ONE. 2016;11:e0146290. doi:10.1371/journal.pone.0146290.

14. Turner CE, Barker-Collo SL, Connell Charlotte JW, et al. Acute hypoxic gas breathing severely impairs cognition and task learning in humans. Physiol Behav. 2015;142:104-10. doi:10.1016/j.physbeh.2015.02.006.

15. Xu L, Wu Y, Zhao T, et al. Effect of high altitude hypoxia on cognitive flexibility. Zhongguo Ying Yong Sheng Li Xue Za Zhi. 2014;30(106-9):118.

16. Yan X. Cognitive impairments at high altitudes and adaptation. High Alt Med Biol. 2014;15:141-5. doi:10.1089/ham.2014.1009.

17. de Aquino Lemos V, Antunes HKM, dos Santos RVT, et al. High altitude exposure impairs sleep patterns, mood, and cognitive functions. Psychophysiology. 2012;49:1298-306. doi:10.1111/j.1469-8986.2012.01411.x.

18. Virues-Ortega J, Buela-Casal G, Garrido E, et al. Neuropsychological functioning associated with high-altitude exposure. Neuropsychol Rev. 2004;14:197-224.

19. Zhang J, Chen R, Wu Y, et al. An EMG study on characteristics of premotor and motor components in an agility reaction time test on athletes. J Sports Med Phys Fit. 2013;53:566-72.

20. Firth PG, Zheng H, Windsor JS, et al. Mortality on Mount Everest, 19212006: descriptive study. BMJ. 2008;337:a2654. doi:10.1136/bmj.a2654.

21. Dykiert D, Hall D, van Gemeren N, et al. The effects of high altitude on choice reaction time mean and intra-individual variability: Results of the Edinburgh Altitude Research Expedition of 2008. Neuropsychology. 2010;24:391.

22. Saugy JJ, Schmitt L, Fallet S, et al. Sleep disordered breathing during live high-train low in normobaric versus hypobaric hypoxia. High Alt Med Biol. 2016;17:233-8. doi:10.1089/ham.2016.0049.

23. Goldstein FC, Zhao L, Steenland K, et al. Inflammation and cognitive functioning in African Americans and Caucasians. Int J Geriatr Psychiatry. 2014;30:934-41. doi:10.1002/gps.4238.

24. Zintl F, Held F. Struktur und Sinn eines modernen Krafttrainings. In: Steinacker JM (ed) Rudern. Berlin: Springer; 1988. p. 197-204.

25. Ainslie PN, Lucas SJ, Burgess KR. Breathing and sleep at high altitude. Respir Physiol Neurobiol. 2013;188:233-56. doi:10.1016/j.resp.2013.05.020.

26. Chung KC, Peisen F, Kogler L, et al. The influence of menstrual cycle and androstadienone on female stress reactions: an fMRI study. Front Hum Neurosci. 2016;10:44. doi:10.3389/fnhum.2016.00044.

27. Clancy KBH, Baerwald AR, Pierson RA, et al. Systemic inflammation is associated with ovarian follicular dynamics during the human menstrual cycle. PLOS ONE. 2013;8:e64807. doi:10.1371/journal.pone.0064807.

28. Roach EB, Bleiberg J, Lathan CE, et al. AltitudeOmics: decreased reaction time after high altitude cognitive testing is a sensitive metric of hypoxic impairment. NeuroReport. 2014;25:814-8. doi:10.1097/ WNR.0000000000000169. 